\title{
How the depth of processing modulates emotional interference - evidence from EEG and pupil diameter data
}

\author{
Marie Luise Schreiter ${ }^{1} \cdot$ Witold X. Chmielewski ${ }^{1} \cdot$ Moritz Mückschel $^{1} \cdot$ Tjalf Ziemssen $^{2} \cdot$ Christian Beste $^{1}$
}

Published online: 12 June 2019

(C) The Psychonomic Society, Inc. 2019

\begin{abstract}
The ability to process emotionally conflicting information is an important requirement for emotional self-control. While it seems obvious that the impact of interfering emotional information critically depends on how deeply this interfering information is processed, it is still unknown what cognitive subprocesses are most affected by manipulating the depth of processing of emotionally interfering information. We examine these aspects integrating neurophysiological (EEG) and source localization data with pupil diameter data as an indirect index of the norepinephrine (NE) system activity. We show that when processing depth of interfering emotional stimulus dimensions is increased, emotional Stroop effects become stronger. The EEG data show that this was associated with modulations of decision-making processes, as reflected by the P3 event-related potential. Notably, the integration with pupil diameter data suggests that these decision processes were modulated by the NE system, especially when the depth of processing of interfering emotional stimulus dimensions was increased. This likely reflects gain modulation processes to facilitate processing of complex interfering, emotional information. The source localization results suggest that regions in the parietal (BA7) and insular cortex (BA13) are associated with these modulatory effects. The results suggest that overcoming more complex emotional interference triggers engagement of the norepinephrine system (indexed by pupil diameter) to facilitate action control mechanisms in a time-specific manner when deeper processing of emotional stimulus dimensions is required.
\end{abstract}

Keywords Emotional conflicts $\cdot$ EEG $\cdot$ Pupil diameter $\cdot$ Norepinephrine system $\cdot$ Stroop

\section{Introduction}

The ability to process relevant emotional information and ignore irrelevant emotional information is central for emotional self-regulation (Berg et al., 2016; Marusak, Martin, Etkin, \& Thomason, 2015; Ochsner \& Gross, 2005). These processes have extensively been examined using emotional interference (Stroop) tasks (Egner, Etkin, Gale, \& Hirsch, 2008; Etkin, Egner, \& Kalisch, 2011; Kunde \& Mauer, 2008; McKenna \&

Electronic supplementary material The online version of this article (https://doi.org/10.3758/s13415-019-00732-0) contains supplementary material, which is available to authorized users.

Christian Beste

christian.beste@uniklinikum-dresden.de

1 Cognitive Neurophysiology, Department of Child and Adolescent Psychiatry, Faculty of Medicine, Technical University of Dresden, Fetscherstrasse 74, 01307 Dresden, Germany

2 MS Centre Dresden, Centre of Clinical Neuroscience, Department of Neurology, Faculty of Medicine, Technical University of Dresden, Dresden, Germany
Sharma, 2004; Pan, Lu, Chen, Wu, \& Li, 2016; Saunders, Milyavskaya, \& Inzlicht, 2015; Schreiter, Chmielewski, \& Beste, 2018b; Zinchenko, Kanske, Obermeier, Schröger, \& Kotz, 2015). In the face-word Stroop task, for example, emotional faces are presented together with an emotional word that is either congruent or incongruent with the presented emotional face (Egner et al., 2008, 2008; Etkin et al., 2011; Kerns et al., 2004; Marusak et al., 2015). Typically, response accuracy and reaction times (RTs) decline when classifying the emotional target dimension (face) on the Stroop stimulus, in trials where the face and the word are incongruent ("emotional Stroop effect") (Algom, Chajut, \& Lev, 2004; MacLeod, 1991; Weissman \& Carp, 2013). Importantly, the emotional Stroop effect, like non-emotional Stroop effects, represents a "stimulus-stimulus conflict" (S-S conflict) (Hommel, 2015). This conflict refers to competition and interference between two stimulus dimensions that are simultaneously presented and are semantically similar (Hommel, 2015). Therefore, the magnitude of emotional (Stroop) effects is likely dependent on how deeply the task-irrelevant and interfering emotional stimulus dimension is processed. Only when both stimulus dimensions are processed deeply, are strong emotional interference effects 
likely to occur. While this seems obvious, an exact characterization of the cognitive-neurophysiological processes being modulated by the depth of processing emotional interfering information is still indeterminate.

In the current study, we examine those aspects integrating neurophysiological and EEG-source localization data with vegetative nervous system data (i.e., pupil diameter data). We experimentally vary the depth of processing (DOP) of the task-irrelevant stimulus dimension by systematically adjusting the intentional relevance of the emotional distractor dimension. Systematically manipulating the DOP of the irrelevant/distracting stimulus dimension should modulate the extent to which emotional conflict is reflected in behavior. Deeper processing of the task-irrelevant stimulus dimensions should result in stronger behavioral Stroop effects, i.e., increased response times and decreased accuracy on incongruent trials. In can therefore be supposed that DOP modulates the difficulty in processing conflicting information.

A natural consequence of a deeper processing, and more difficult processing of the interfering information, is that conflict-monitoring processes and the decision about which response to select become complicated. Considering these processes, it has been suggested that the norepinephrine (NE) system strongly modulates these processes. The adaptive gain theory suggests that the locus coeruleus-norepinephrine (LC-NE) system modulates neural processing during task-relevant decisions (Aston-Jones \& Cohen, 2005). It has been suggested that the phasic release of NE facilitates decision processes under more control-demanding circumstances and optimizes behavioral performance according to the relevant task at hand (AstonJones \& Cohen, 2005). Therefore, it is likely that variations in the DOP of emotional interfering information affect the modulation of task-relevant decision points by the NE system. Importantly, it has been shown that the NE system activity modulates human pupil diameter in a process- and time pointspecific manner ( Costa \& Rudebeck, 2016; Joshi, Li, Kalwani, \& Gold, 2016; Varazzani, San-Galli, Gilardeau, \& Bouret, 2015). Therefore, the pupil diameter can be used as an indirect index of NE system activity (Dippel, Mückschel, Ziemssen, \& Beste, 2017; Helton et al., 2005; Hong, Walz, \& Sajda, 2014; Hou, Freeman, Langley, Szabadi, \& Bradshaw, 2005; Jepma \& Nieuwenhuis, 2011; Mückschel, Chmielewski, Ziemssen, \& Beste, 2017a; Murphy, Robertson, Balsters, \& O'connell, 2011). The pupil diameter is modulated by tonic and phasic NE activity (Hong et al., 2014; Hou et al., 2005; Jepma \& Nieuwenhuis, 2011; Murphy et al., 2011). Larger pupil diameters have been shown to reflect higher NE concentrations (Hou, Freeman, Langley, Szabadi, \& Bradshaw, 2005b; Phillips, Szabadi, \& Bradshaw, 2000). Recent data show that neurophysiological indices of response selection and cognitive control are modulated by NE system activity, as indexed using pupil diameter (Chmielewski, Mückschel, Ziemssen, \& Beste, 2017; Dippel et al., 2017; Mückschel, Chmielewski, et al., 2017; Wolff, Mückschel, Ziemssen, \& Beste, 2018); i.e., there are correlations between the pupil diameter data and the neurophysiological (EEG) data at a specific time point in the processing cascade. With regard to the DOP manipulation in the current study, we hypothesize that there should be strong correlations between pupil diameter data and neurophysiological data when the DOP of the irrelevant stimulus dimension is increased. This is because task-relevant decision processes become more demanding when irrelevant, distracting emotional information in processed in depth. No or much smaller correlations should be evident when the irrelevant stimulus dimension is not processed in depth. As mentioned, modulations of the NE system during cognitive control tasks occur at specific time points. It has been shown that processes in the N2 ERP, and even more so in the P3 ERP time window, are modulated by the NE system as indexed by the pupil diameter (Chmielewski et al., 2017; Dippel et al., 2017; Mückschel, Chmielewski, et al., 2017; Wolff et al., 2018). This is in line with other studies (Murphy et al., 2011; Nieuwenhuis et al., 2005; Warren \& Holroyd, 2012). In particular, neural activity in the P3 time window has long been argued to be strongly modulated by the NE system and may even reflect NE activity (Nieuwenhuis et al., 2005). This is also plausible because the P3 component reflects decision making and stimulus-response association processes (Gray, Ambady, Lowenthal, \& Deldin, 2004; Twomey, Murphy, Kelly, \& O'Connell, 2015), which are known to be modulated by the NE system (Aston-Jones $\&$ Cohen, 2005). These processes are enhanced when interfering information has a strong impact on information processing (Mennes, Wouters, Bergh, Lagae, \& Stiers, 2008). Thus, it is reasonable to expect that in the DOP-condition the increased complexity of decision-making processes should evoke greater involvement of the NE system activity reflected in pupil diameter. We hypothesize that the pupil diameter is correlated with neurophysiological data at the time point of the P3 ERP in the DOP group, but not in the group in which the interfering emotional information is not processed in depth. We also hypothesize that modulations of the DOP will affect the amplitude of the P3 ERP-component. Notably, it is well established that pupil dilation occurs with a $200-$ to $500-\mathrm{ms}$ delay relative to LC-NE activity (Joshi et al., 2016; Nieuwenhuis, De Geus, \& Aston-Jones, 2011). So, correlations between pupil reaction and neurophysiological signals corresponding to the P3 time window should be visible within a time frame of around $700 \mathrm{~ms}$ onwards. During emotional Stroop conflicts, it is well known that the P3 amplitudes are increased in emotionally congruent compared to incongruent trials (Clayson \& Larson, 2013; Delplanque, Lavoie, Hot, Silvert, \& Sequeira, 2004; Larson, Clayson, \& Clawson, 2014; Schreiter et al., 2018b; Yang et al., 2013). Since an increased DOP is hypothesized to increase emotional Stroop effects, we also hypothesize that the P3 modulations between congruent and incongruent trials are also stronger when DOP is increased. From a functional 
neuroanatomical perspective, these effects are likely to be associated with activation differences in parietal regions, but in addition, the insular cortex likely plays an important role. Parietal regions have frequently been associated with modulations in the P3 ERP-component (Bledowski et al., 2004; Gray et al., 2004) and also the NE system modulates P3-related processes via parietal regions (Wolff et al., 2018). The insular cortex may be relevant because this region plays a major role in the interpretation of emotional information and has been shown to be involved in emotional and non-emotional interference processing (Gogolla, 2017; Simmons, Matthews, Paulus, \& Stein, 2008; Xue et al., 2016). Importantly, it has been shown to modulate emotional decision-making processes by orchestrating external emotional with internal physiological signals (such as NE release) to predict future decision outcomes particularly in situations of affective ambiguity (Gogolla, 2017). Therefore, we hypothesize that modulations of the $P 3$ ERP-component that are modulated by the NE system are associated with the insula. Specifically, we hypothesize that there is stronger insular and parietal activity within the DOP group.

Since previous findings suggest that emotional stimuli have identical effects on early attentional selection processes (Brosch, Sander, Pourtois, \& Scherer, 2008; Clayson \& Larson, 2013; Pool, Brosch, Delplanque, \& Sander, 2016) we do not expect effects of DOP manipulation on P1 and N1 ERPs. Considering the resolution of interferences, the N2 ERP-component is more negative during conflicting trials than on non-conflicting/non-interfering trials (Chen et al., 2016; Chmielewski \& Beste, 2017; Larson et al., 2014; Shang, Fu, Qiu, \& Ma, 2016). However, due to the complexity of stimuli in word-face emotional Stroop tasks, no modulations of the N2 by interference effects have been observed (Schreiter, Chmielewski, \& Beste, 2018a; Schreiter et al., 2018b). Therefore, we do not expect to observe amplitude modulations and differential correlations with the pupil diameter in the $\mathrm{N} 2$ time window. The CSP (conflict-slow potential) has been shown to be modulated by emotional Stroop conflict (Chen et al., 2016; Larson et al., 2014; Ma, Liu, Zhong, Wang, \& Chen, 2014; Schreiter et al., 2018b), but seems to reflect pro-active control processes rather than conflict control in response to complex task-demands (Larson et al., 2014; Pan et al., 2016; Shen, Xue, Wang, \& Qiu, 2013). Therefore, it is unlikely that there are modulations of the processes by the NE system (i.e., correlations with pupil diameter data) and modulations in amplitude due to a manipulation of DOP.

\section{Materials and methods}

\section{Participants}

We tested two groups of participants. Each group included $\mathrm{N}=20$ healthy participants between 18 and 30 years of age (mean age $23.73 \pm 0.72$ years; $\mathrm{n}=12$ females in each group). In one group, the depth of processing of the interfering emotional stimulus was increased (refer to the section Manipulation of depth of processing (DOP)). The sample sizes used for each group have been shown to yield reliable correlations between pupil diameter and ERP-data (Dippel et al., 2017; Mückschel, Chmielewski, et al., 2017; Wolff et al., 2018). The reason for choosing a between-subject and not a within-subject design is that "order effects" can never be ruled out using within-subject designs. A crossover design (i.e., where the order of DOP vs. non-DOP is counterbalanced across participants) requires testing, whether "test order" could have affected the results. The consequence is that a between-subject factor is still needed for this kind of investigation. Relating to this aspect, learning/neural plasticity processes of stimulus features and gain-control mechanisms (refer to actions of the NE system and the recording of the pupil diameter data) are closely related, as learning also enhances the neural signal-to-noise ratio in cortical circuits (Dosher \& Lu, 1998). This is particularly important when assessing processes related to stimulus-stimulus conflicts.

All participants had normal or corrected-to-normal vision and were screened on personal health background to ensure our sample was free of individuals previously diagnosed with any psychiatric or neurological disorders, or taking regular medication. All participants provided written informed consent prior to the experiment, and received payment $(15 €)$ or course credits (TU Dresden students) after completion of the study. The study was approved by the institutional review board of the Medical Faculty of the TU Dresden and conducted in accordance with the Declaration of Helsinki.

\section{Task}

We employed an emotional Stroop paradigm in which emotional interference is induced by the incongruency between two emotional dimensions of one presented stimulus (emotional faces and words). Pictures of emotional facial expressions represented the task-relevant dimension, while emotional words printed across the picture constituted the task-irrelevant distractor dimension. We used pictures from four different Caucasian males depicting either a happy or an angry facial expression. The pictures were selected from the NimStim, MacBrain Face Stimulus Set (Nim Tottenham supported by the John D. and Catherine T. MacArthur Foundation Research Network on Early Experience and Brain Development, open source available on http://www.macbrain. org/resources.htm). The faces were presented in gray-scale using XNView (for windows) and congruent or incongruent emotional words, i.e., "FREUDE" or "ÄRGER" (German for "HAPPINESS" and "ANGER") were written centrally across the faces in red capital letters (refer to Fig. 1 for details).

There were equal proportions of congruent and incongruent stimuli for each emotion, adding up to 32 different stimuli. 


\section{a}

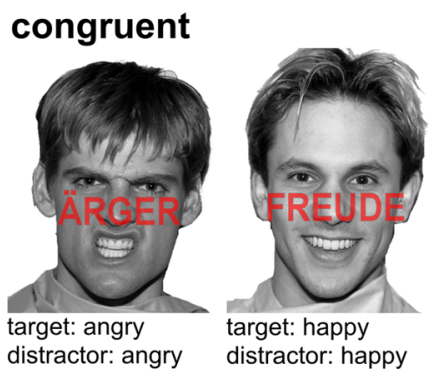

incongruent
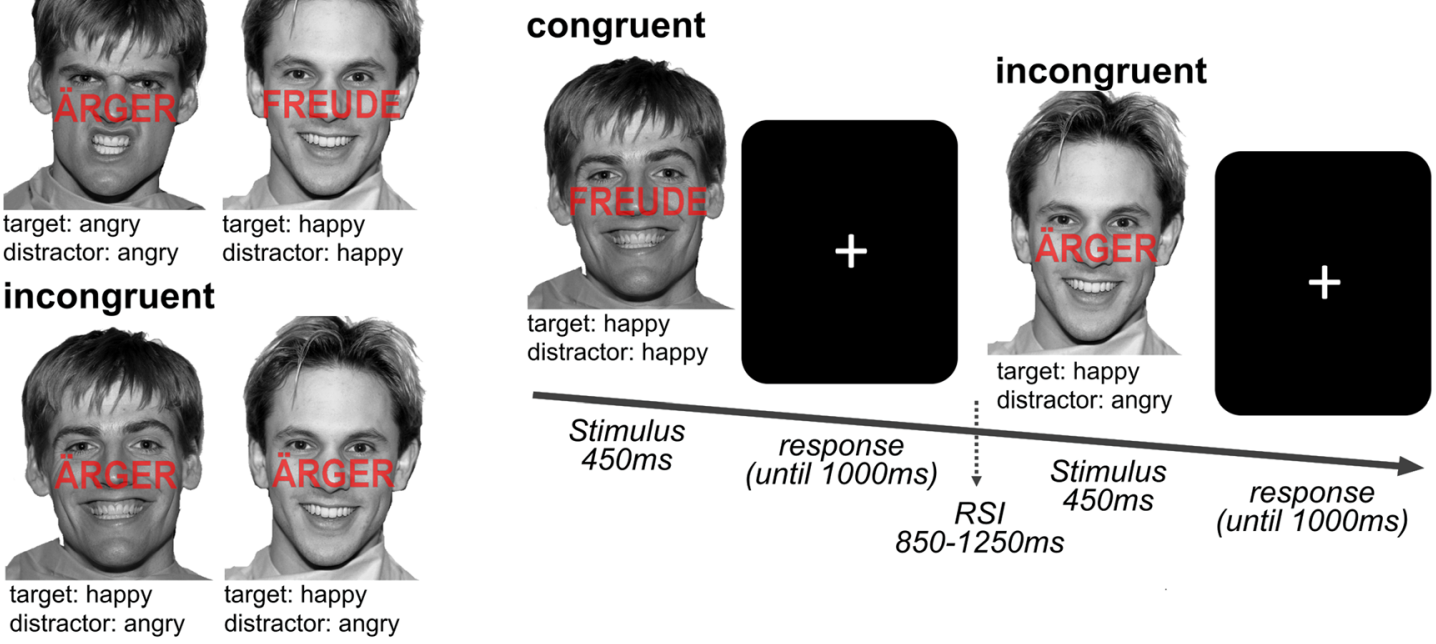

b

DOP - GROUP

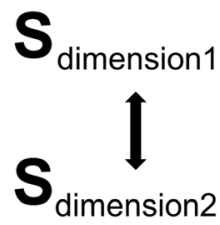

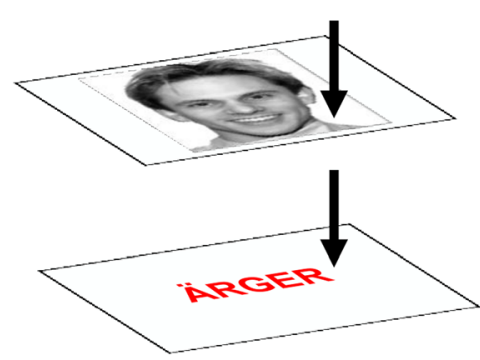

CONTROLS

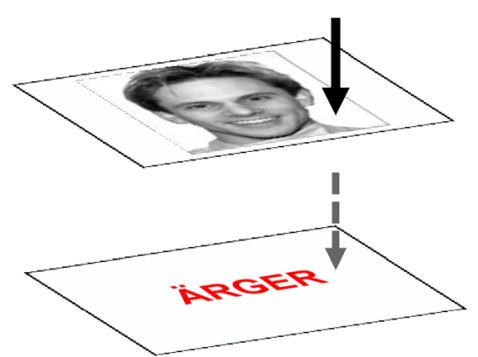

Fig. 1 Example stimuli and experimental paradigm of the emotional Stroop task and depiction of the conceptual background of the current investigation. (a) Participants were instructed to classify the task-relevant emotional expression of the face (i.e., angry or happy) that had a taskirrelevant emotional word (either angry or happy) written across. Stimuli were either congruent or incongruent with respect to facial expression (emotional target: stimulus dimension 1) and word (emotional distractor: stimulus dimension 2), which created emotional interference. Emotional interference effects were greater in the DOP group, where the distracting stimulus dimension 2 (b) required deeper levels of processing due to intermittent queries. In the DOP condition, both aspects of the composite stimuli are processes, whereas it is possible to ignore the written word (i.e., dimension 2) of the stimuli
These stimuli were pseudo-randomly presented in four blocks of 216 trails each (i.e., 864 trials in total). In each block, the same number of congruent and incongruent stimuli were presented and it was ensured that the same proportion of the different stimuli was presented. Both experimental groups were asked to respond via the arrow keys of a conventional computer keyboard. The stimuli (face-target/task-relevant dimension and emotional-word/task-irrelevant dimension) were presented for $450 \mathrm{~ms}$ and then followed by the presentation of the fixation cross for the rest of the trial. All participants were instructed to identify the correct facial emotional expression as quickly as possible for each trial, and encouraged to make use of the allocated breaks if needed. For stimuli depicting happy target emotions participants were required to press the left ctrlkey, and for angry emotions the right ctrl-key. If participants did not respond within 1,000 ms of target onset, the trial was treated as a miss. After the response was executed, a variable response stimulus interval (RSI), jittered between 850 and $1,250 \mathrm{~ms}$, was included, in which a central fixation cross was presented. Likewise, responses to intermittent queries within the "depth of processing" group were given with the left and right ctrl-keys (left- happy/right- angry). All participants were instructed not to double-press a button and to avoid missing responses as much as possible. Before commencing with the experiment, participants were able to become familiar with the experimental design by conducting a 40 -trial exercise. In total, the task lasted for approximately $40 \mathrm{~min}$.

\section{Manipulation of depth of processing (DOP)}

We systematically enhanced the depth in which the taskirrelevant emotional stimulus dimensions had to be processed by adding intermittent queries about the content of the emotional distractor dimension in one group of participants (DOP 
group). One group of participants was intermittently presented with infrequent queries about the emotional valence of the distractor (word) stimulus in the trial immediately preceding that query (i.e., "Please indicate which word was presented to you in the previous trial? Press the left key for 'ANGER' or right key for 'HAPPY'.”). The queries were pseudorandomized and equally distributed across all experimental blocks. This was done to direct participants' attention to the distractor dimension (i.e., enhance the intentional relevance of the task-irrelevant emotional information). The participants were thus forced to process the emotional face and the emotional word in depth. Queries were treated as hits if the correct answer was obtained within 300-1,300 ms.

\section{EEG recordings and analyses}

EEG data were recorded with a sampling rate of $500 \mathrm{~Hz}$ using a 60-channel system (BrainAmp, Brain Products Inc.). Passive $\mathrm{Ag} / \mathrm{AgCl}$-electrodes (60 recording electrodes) were mounted in an elastic cap (EasyCap Inc.) and arranged in equidistant positions approximating the positions of the $10 / 20$ system. The ground and reference electrode were placed at coordinates theta $=58$, phi $=78$, and theta $=90$, phi $=90$, respectively. Electrode impedances were kept below $5 \mathrm{k} \Omega$. Subsequent data analysis was performed in the Brain Vision Analyzer 2 software package (BrainProducts, Inc.): Offline, a band pass filter of 0.5 $20 \mathrm{~Hz}$ (with a slope of $48 \mathrm{~dB} /$ oct each) and a notch filter of $50 \mathrm{~Hz}$ were applied. In the software package used to analyze the ERP data, even the sharp filter setting with a slope of 48 $\mathrm{dB} /$ oct cannot exclude residual $50 \mathrm{~Hz}$ activity still being evident in the data. This can be seen when running an FFT of the data. Therefore, the $50-\mathrm{Hz}$ notch filter is applied to eliminate even the residual $50-\mathrm{Hz}$ contamination. It needs to be noted that the results reported below remained the same when a less steep filter slope (e.g., $16 \mathrm{db} / \mathrm{oct}$ ) was used. Following that, raw data inspection was conducted manually to reject technical artifacts from the EEG. Then, an independent component analysis (ICA; Infomax algorithm) was conducted to remove recurrent artifacts. ICA components revealing horizontal and vertical eye movements, blinks, and pulse artifacts were manually discarded (number of discarded ICs: $4 \pm 1.5$ ). The data were then segmented for each emotional condition (angry, happy) for congruent as well as incongruent trials. Trials were only taken into account when the correct response was given within $1,000 \mathrm{~ms}$ of target onset. The segmentations were locked to target stimuli starting $250 \mathrm{~ms}$ prior to target onset and finishing $1,000 \mathrm{~ms}$ after its onset. Afterwards, an automated artifact rejection was applied for all the segments. Activity below $0.5 \mu \mathrm{V}$ in a 100-ms period and a maximal value difference of $200 \mu \mathrm{V}$ in $200 \mathrm{~ms}$ within the epoch were used as rejection criteria. If an artifact was detected in a trial, the trial was discarded. Overall, $\sim 1.6 \%$ of trials were discarded due to these issues. To eliminate the reference potential from the data and to re-reference the data, we applied a current source density (CSD) transformation (Kayser \& Tenke, 2015; Nunez \& Pilgreen, 1991), which also serves as a spatial filter resulting in values for amplitudes in $\mu \mathrm{V} / \mathrm{m}^{2}$. A baseline correction from $-200 \mathrm{~ms}$ to 0 prior to target onset was applied and the relevant ERP components, P1 (at P7 and P8: after target presentation onset), N1 (at P7 and P8: 140 $180 \mathrm{~ms}$ ), N2 (at Cz: 220-240 ms), P3 (at C4: 550-660 ms), and CSP (at FCz: 430-470 ms) were identified by means of scalp topography. Within these search intervals, a semi-automatic peak detection was conducted for the ERP components P1, $\mathrm{N} 1$, and N2. For the P3 and the CSP an area export was conducted for each corresponding search interval. The choice of these search intervals for ERP quantification was validated using a statistical approach outlined in Mückschel et al. (2014). In so doing, the above time intervals were taken and the mean amplitude within the defined search intervals was determined for each of the 60 electrode positions. Then, to compare each electrode against an average of all other electrodes, Bonferroni correction for multiple comparisons (critical threshold, $\mathrm{p}=.0007$ ) was used. Only electrodes that displayed significantly larger mean amplitudes (i.e., negative for the $\mathrm{N}$ potentials and positive for the P-potentials) when compared to other electrodes were chosen. This procedure revealed the same electrodes as those chosen by visual inspection.

For the source localization analyses, we used sLORETA (standardized low resolution brain electromagnetic tomography) (Pascual-Marqui, 2002), which provides a single solution to the inverse problem (Marco-Pallarés, Grau, \& Ruffini, 2005; Pascual-Marqui, 2002). The intracerebral volume is partitioned into 6,239 voxels at $5-\mathrm{mm}$ spatial resolution. Then, the standardized current density at each voxel is calculated in a realistic head based on the MNI152 template. There is mathematical evidence that SLORETA provides reliable results without a localization bias (Sekihara, Sahani, \& Nagarajan, 2005). Moreover, there is evidence from EEG/ fMRI and neuro-navigated EEG/TMS studies underlining the validity of the sources estimated using sLORETA (Dippel \& Beste, 2015; Hoffmann, Labrenz, Themann, Wascher, \& Beste, 2014). The voxel-based sLORETA images were compared between groups and experimental conditions using the sLORETA-built-in voxel-wise randomization tests with 2,000 permutations, based on statistical nonparametric mapping (SnPM). Voxels with significant differences $(\mathrm{p}<$ .01 , corrected for multiple comparisons) between contrasted conditions and groups were located in the MNI-brain.

\section{Pupil diameter recording and analysis}

Pupil diameter recordings and analysis were conducted using established protocols (Dippel et al., 2017; Mückschel, Chmielewski, et al., 2017; Mückschel, Gohil, Ziemssen, \& Beste, 2017b; Wolff et al., 2018). The pupil diameter was recorded using a RED 500 eye-tracking device and the 
software iView X (RED 500 eye-tracking device and the software iView X (SensoMotoric Instruments $\mathrm{GmbH}$ ) at a $500-\mathrm{Hz}$ sampling rate. Before the start of the experiment, the device was calibrated using a 9-point calibration. Eye blinks were automatically interpolated by the eye-tracking recording software. For all subjects, the mean pupil diameter of both eyes was used for data analysis. The pupil diameter did not differ between the left and right eye $(p>.95)$ and the diameter of the left eye was strongly correlated with that of the right eye $(\mathrm{r}$ $>.96 ; p<.001)$. The pupil diameter data and EEG data were synchronized using the EYE-EEG extension (Dimigen, Sommer, Hohlfeld, Jacobs, \& Kliegl, 2011) for EEGLab (Delorme \& Makeig, 2004), (http://www2.hu-berlin.de/ eyetracking-eeg) run on MATLAB (Mathworks, Inc., Natick, MA, USA). For data analysis, the pupil diameter data were low-pass filtered (IIR filter with $20 \mathrm{~Hz}$ at a slope of $48 \mathrm{~dB} /$ oct each). Remaining artifacts (e.g., blink artifacts) were manually removed by means of raw data inspection. Segmentation and baseline correction were conducted the same way as the EEG data. To integrate the pupil diameter and EEG data we used Pearson correlations between the pupil diameter data and the ERP data. For this, the segments were split into time bins of approximately $2 \mathrm{~ms}$ (i.e., the length of each sampling point for a sampling rate of $500 \mathrm{~Hz}$ ). For the integration of EEG and pupil diameter data, every EEG data time bin was correlated with every pupil diameter data time bin across all participants. Since there is a low signal-to-noise ratio in the single-trial ERP data, no within-subject correlations were calculated according to recent protocols (Chmielewski et al., 2017; Mückschel, Gohil, et al., 2017).

\section{Statistical analysis}

Correct responses (\%) and RTs were analyzed using mixedeffects ANOVAs using the within-subject factors "congruency" (congruent vs. incongruent) and "emotion" (happy and angry) and the between-subject factor "group" (DOP/controls). For the neurophysiological data, the factor "electrode" (for P1 and N1) was added in the model. Greenhouse-Geisser correction was applied where appropriate and post hoc tests were Bonferroni-corrected.

\section{Results}

\section{Behavioral data}

\section{Accuracy data}

The descriptive behavioral data for both groups, i.e. mean RTs $(\mathrm{ms})$ and accuracy $(\%)$ for the congruency conditions is shown in Fig. 2, including the standard error of the mean (SEM).
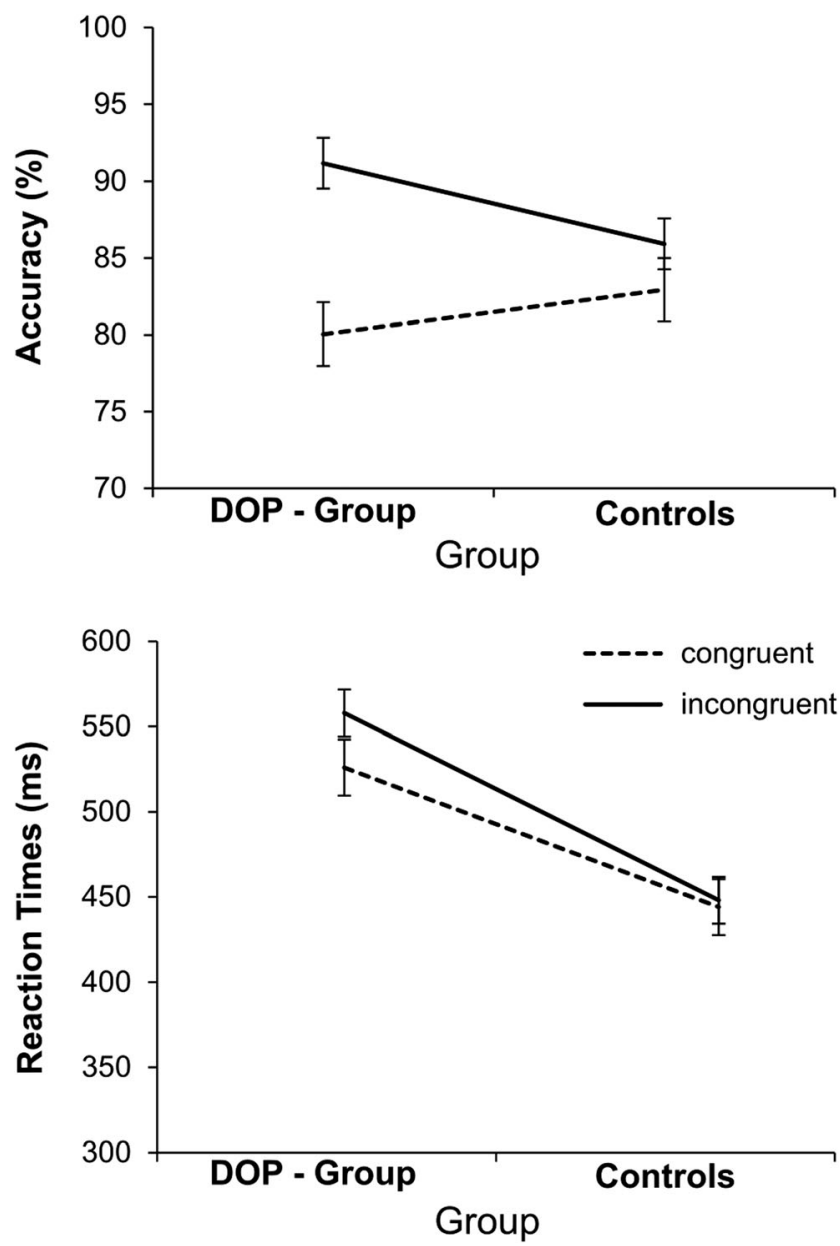

Fig. 2 Summary of the behavioral results. (a) Accuracy (\%) and (b) reaction times (RTs) $(\mathrm{ms})$ for each group (DOP group and controls), data (mean $\pm \mathrm{SEM}$ ) are for congruent and incongruent emotional trials. Incongruent trials elicited slower RTs and less accurate responses than congruent trials. Emotional interference effects were greater within the DOP group, where the distracting stimulus dimension 2 required deeper levels of processing due to intermittent queries

With regard to accuracy, the mixed-effects ANOVA revealed a main effect of "congruency," $(\mathrm{F}(1,38)=47.06$; $\mathrm{p}<$ $\left..001 ; \eta_{\mathrm{p}}{ }^{2}=.553\right)$. Overall, participants' performance was significantly more accurate on congruent trials $(88.56 \% \pm 1.17)$ than on incongruent trials $(81.50 \% \pm 1.46)$. Furthermore, there was a main effect of "emotion" $\left(\mathrm{F}(1,38)=11.35 ; \mathrm{p}<.002 ; \eta_{\mathrm{p}}{ }^{2}\right.$ $=.230$ ), showing that performance on happy target emotions $(86.70 \% \pm 1.14)$ was more accurate compared to angry target emotions $(83.35 \% \pm 1.49)$ (refer to Fig. 1 in the Electronic Supplemental Material). Most importantly, there was a significant interaction "congruency $\times$ group" $(\mathrm{F}(1,38)=15.69 ; \mathrm{p}<$ $\left..001 ; \eta_{\mathrm{p}}{ }^{2}=.292\right)$. To further analyze this interaction, we calculated the average hit rate values for each group for congruent as well as incongruent trials (pooled across emotion conditions): Independent-samples t-tests revealed that hit rates in the DOP group on congruent trials $(91.19 \% \pm 1.35)$ were significantly higher compared to hit rates on congruent trials 
$(85.92 \% \pm 1.92)$ in the control group, $(\mathrm{t}(38)=-2.24, \mathrm{p}<.05)$. Hit rates in the DOP group on incongruent trials $(82.94 \% \pm$ 1.67) compared to hit rates on incongruent trials $(80.05 \% \pm$ $2.21)$ in the control group did not significantly differ $(\mathrm{t}(38)=$ $.99, p=.33$ ). However, since we were focused on the effects of depth of processing on conflict effects (i.e., performance differences between congruent and incongruent trials), we compared hit-rate differences within each group.

Post hoc paired t-tests for each group revealed that hit rates within the DOP group on congruent trials $(91.19 \% \pm 1.35)$ were significantly higher than on incongruent trials $(80.05 \%$ $\pm 2.21) ;(\mathrm{t}(19)=-6.86, \mathrm{p}<.001)$. Likewise, for the control group, hit rates on congruent trials $(85.92 \% \pm 1.92)$ were significantly more accurate than on incongruent trials (82.94 $\% \pm 1.67) ;(\mathrm{t}(19)=-2.36, \mathrm{p}<.05)$, but the size of this effect was smaller in the control than in the DOP group (refer to the $t-$ values). This shows that the interaction is driven by the DOP group. There were no other main or interaction effects (all $\mathrm{F} \leq$ $1.89 ; \mathrm{p} \geq .378)$.

\section{Reaction times}

With regard to the RTs, the mixed-effects ANOVA revealed a main effect of "congruency", $\left(\mathrm{F}(1,38)=22.48 ; \mathrm{p}<.001 ; \eta_{\mathrm{p}}{ }^{2}=\right.$ .372). Overall, participant's reactions was significantly faster on congruent trails $(485 \mathrm{~ms} \pm 10)$ than on incongruent trials (503 $\mathrm{ms} \pm 12$ ). Furthermore, there was a main effect of "emotion" $\left(\mathrm{F}(1,38)=12.89 ; \mathrm{p}=.001 ; \eta_{\mathrm{p}}{ }^{2}=.253\right)$ showing that responses to happy target emotions $(490 \mathrm{~ms} \pm 11)$ were faster than to angry target emotions (499. ms \pm 11 ) (refer to Fig. 2 in the Electronic Supplemental Material). There was a significant interaction "congruency $\times$ group" $(\mathrm{F}(1,38)=$ $12.60 ; \mathrm{p}<.001 ; \eta_{\mathrm{p}}^{2}=.253$ ).

To further analyze the congruency $\times$ group interaction, we calculated the average RTs for each group for congruent as well as incongruent trials (pooled across emotion condition). Independent-samples t-tests revealed that RTs in the DOP group on congruent trials (527 $\mathrm{ms} \pm 15$ ) were significantly slower compared to RTs on congruent trials $(444 \mathrm{~ms} \pm 11)$ in the control group, $(\mathrm{t}(38)=-4.23, \mathrm{p}<.001)$. Likewise, RTs in the DOP group on incongruent trials $(559 \mathrm{~ms} \pm 20)$ were significantly slower compared to RTs on incongruent trials $(449 \mathrm{~ms} \pm 12)$ in the control group $(\mathrm{t}(38)=-4.71, \mathrm{p}<.001)$. Since the main aim was to investigate the effects of manipulating depth of processing on emotional Stroop effects (i.e., performance differences between congruent and incongruent trials), we compared RT differences within each group. Post hoc paired t-test comparison for each group revealed that responses in the DOP group on congruent trials $(527 \mathrm{~ms} \pm 15)$ were significantly faster than on incongruent trials $(559 \mathrm{~ms} \pm$ $20) ; \mathrm{t}(19)=-4.37, \mathrm{p}<.001)$. For the control group, RTs on congruent trials $(444 \mathrm{~ms} \pm 11)$ and incongruent trials $(449 \mathrm{~ms}$ \pm 12 ) did not significantly differ, $\mathrm{t}(19)=-1.88, \mathrm{p}=.074$ ).
There were no other main or interaction effects, (all $\mathrm{F} \leq$ $1.29 ; \mathrm{p} \geq .417)$.

\section{Neurophysiological data}

As our behavioral data showed that the important interaction of "congruency $\times$ group" was not further modulated by emotion and there was no interaction of "emotion $\times$ group", the factor emotion (i.e., positive or negative) does not modulate the pattern of results. Hence, we did not analyze this factor for the EEG data. Any effects that may have occurred in the EEG data are not possible to interpret and reflect epiphenomena due to the lack of behavioral importance.

The P1 and N1 ERP-components are shown in Fig. 3. The N2 ERP-component is shown in Fig. 4a, the CSP ERPcomponent in Fig. $4 b$.

With regard to the $\mathrm{P} 1$, we found a significant main effect of "electrode" $(\mathrm{P} 8 / \mathrm{P} 7),\left(\mathrm{F}(1,38)=13.55 ; \mathrm{p}<.000 ; \eta_{\mathrm{p}}{ }^{2}=.263\right)$ in the mixed-effects ANOVA, showing the P1 amplitude to be increased at the electrode P8 $\left(49.52 \mu \mathrm{V} / \mathrm{m}^{2} \pm 3.41\right)$ in comparison to $\mathrm{P} 7\left(37.21 \mu \mathrm{V} / \mathrm{m}^{2} \pm .3 .25\right)$. No other significant main effects or interactions were observed (all $\mathrm{F} \leq 2.83 ; \mathrm{p} \geq$ .075). With regard to the repeated-measures ANOVA for N1 amplitudes, there were no significant main or interaction effects (all $F \leq 1.50 ; p \geq .229$ ). Similarly, in the repeatedmeasures ANOVA for the $\mathrm{N} 2$ amplitudes (at electrode $\mathrm{Cz}$ ), there were neither main nor interaction effects (all $\mathrm{F} \leq 1.31$; $\mathrm{p}$ $\geq .259$ ). For the CSP component (at electrode FCz), mixedeffects ANOVA did not reveal any significant main or interaction effects (all $\mathrm{F} \leq 0.82 ; \mathrm{p} \geq 507$ ).

The P3 ERP-component is shown in Fig. 5.

In the mixed-effects ANOVA for the P3 ERP-component (at electrode C4), a main effect of congruency was observed $\left(\mathrm{F}(1,38)=11.77 ; \mathrm{p}=.001 ; \eta_{\mathrm{p}}{ }^{2}=.241\right)$, showing P3 amplitudes to be increased on congruent trials $\left(16.73 \mu \mathrm{V} / \mathrm{m}^{2} \pm 1.49\right)$ in comparison to incongruent trials $\left(15.51 \mu \mathrm{V} / \mathrm{m}^{2} \pm 1.41\right)$. Moreover a "congruency $\times$ group" interaction was observed $\left(F(1,38)=8.86 ; p=.005 ; \eta_{p}{ }^{2}=.193\right)$. Independent-samples $t$ tests revealed that on congruent trials there was no significant difference between average P3 in the DOP group $(13.56 \mu \mathrm{V} /$ $\left.\mathrm{m}^{2} \pm 2.95\right)$ compared to congruent trials $\left(17.54 \mu \mathrm{V} / \mathrm{m}^{2} \pm 2.31\right)$ in the control group. In incongruent trials $\mathrm{P} 3$ amplitude in the DOP group $\left(11.00 \mu \mathrm{V} / \mathrm{m}^{2} \pm 3.21\right)$ also did not significantly differ from P3 amplitude within the control group $(17.68 \mu \mathrm{V} /$ $\mathrm{m}^{2} \pm 2.01$ ), (all $\mathrm{t} \geq 1.67 ; \mathrm{p} \geq .103$ ).

For the DOP group, post hoc paired t-tests showed that average $\mathrm{P} 3$ amplitudes significantly differed between congruent $\left(13.56 \mu \mathrm{V} / \mathrm{m}^{2} \pm 2.95\right)$ and incongruent trials $\left(11.00 \mu \mathrm{V} / \mathrm{m}^{2}\right.$ $\pm 3.21)(\mathrm{t}(19)=4.13, \mathrm{p}=.001)$. For the control group, there was no significant difference in $\mathrm{P} 3$ amplitude between congruent $\left(17.54 \mu \mathrm{V} / \mathrm{m}^{2} \pm 2.31\right)$ and incongruent trials (17.68 $\left.\mu \mathrm{V} / \mathrm{m}^{2} \pm 2.01\right),(\mathrm{t}(19)=.409, \mathrm{p}=.687)$. 


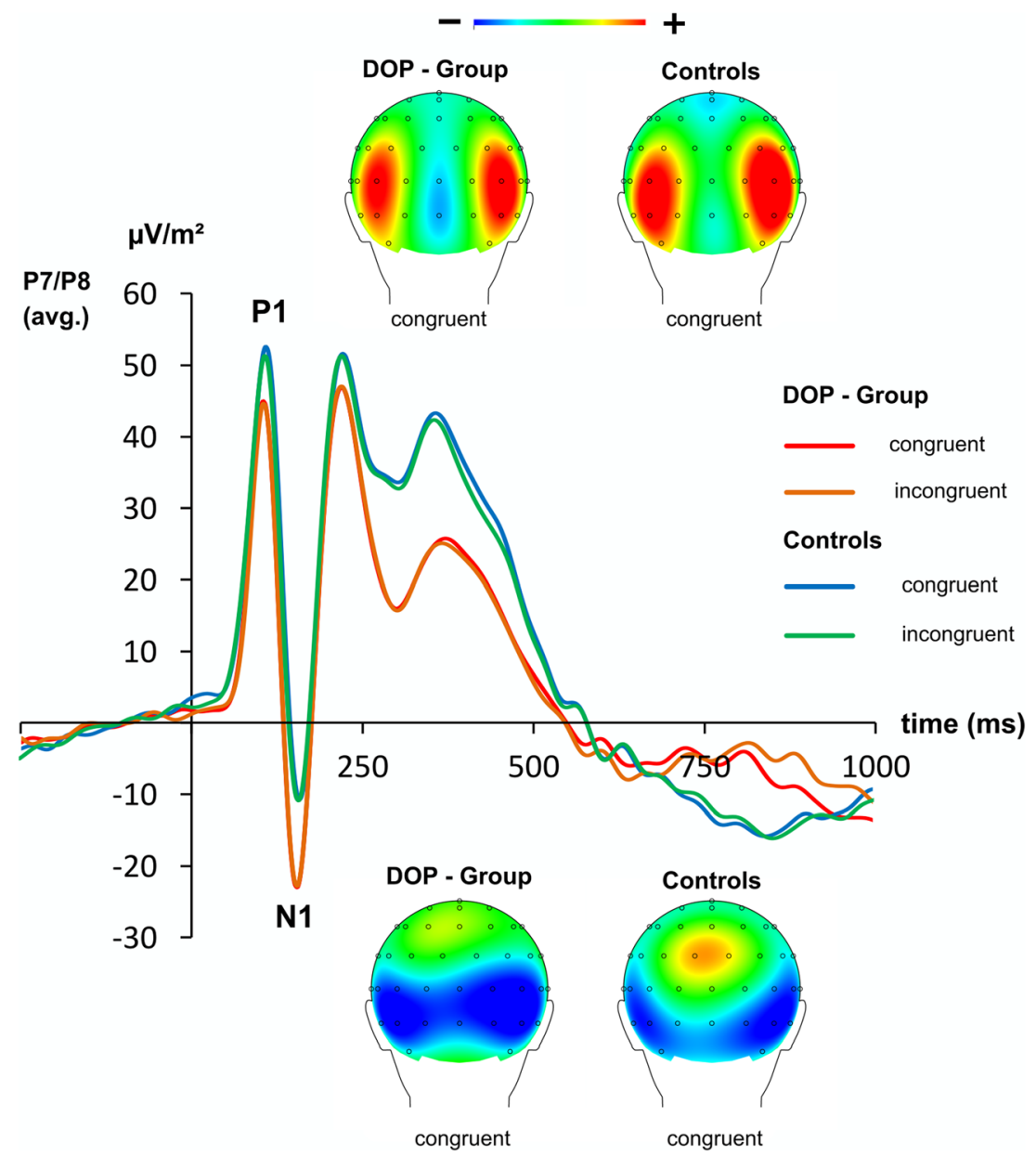

Fig. 3 P1 and N1 ERP components shown for electrode P7/P8 and representative topography maps of the scalp electrical potential for P1 and $\mathrm{N} 1$ at the peak of each ERP-component for each group (DOP

group and controls). In the topographies, red denotes positivity, and blue negativity. The lines represent congruent emotional trials. Time point zero denotes onset of the target stimulus

\section{Pupil diameter integration with EEG}

As can be seen in Fig. 6a, pupil dilation differed between the groups for a time range around $500-700 \mathrm{~ms}$ after stimulus onset. Mixed-effects ANOVA for the pupil dilation data revealed no main or interaction effects (all $\mathrm{F} \leq 3.00 ; \mathrm{p} \geq .091$ ). The results from the correlation analysis of the pupil diameter data with the ERP data are shown in Fig. 6b.

Positive correlations are shown in hot colors, negative correlations are shown in cold colors. Only significant correlations are shown. As can be seen in Fig. 6b, there was a significant positive correlation of the pupil diameter data within the time range between 250 and $300 \mathrm{~ms}$ after target stimulus presentation and the ERP time-series data with the time range between 700 and $1,000 \mathrm{~ms}$ after target presentation in the DOP group ( $\mathrm{r}$ $>.57 ; \mathrm{R}^{2}=.32 ; \mathrm{p}<.01$ ) at electrode $\mathrm{C} 4$, which was shown to reveal modulations in the $\mathrm{P} 3$ amplitudes. Notably, no significant correlations were obtained for the control group (all $\mathrm{r}<$ $-.2 ; \mathrm{R}^{2}=.04 ; \mathrm{p}>.4$ ), in which the depth of processing was not experimentally increased. Using sLORETA, we examined which brain structures were activated in the time period showing the correlations between pupil diameter and ERP activity in the DOP group. Thus, we took the time range from 700 $1,000 \mathrm{~ms}$ after target stimulus and compared the mean activity in this time window against zero. The results reveal that areas in the superior parietal cortex (BA7) and the insular cortex (BA13) were associated with modulations in neurophysiological activity in this time window (see Fig. 6b).

The specificity of effects is further underlined by the correlation analyses using electrodes P7/P8 (see Fig. 6c), which also revealed no significant correlations between the pupil diameter data and the ERP data (all $\mathrm{r}<-.2 ; \mathrm{R}^{2}=.04 ; \mathrm{p}>.4$ ) in the above time window where correlations between pupil diameter data and the EEG data were evident and whereo an interaction "congruency $\times$ group" was also obtained. As can be seen in Fig. 6c, there are some significant correlations between pupil diameter data and EEG data at electrodes P7/ P8. Considering that pupil reactions occur with an approximate delay of about $200 \mathrm{~ms}$ relative to LC-NE activity (Dippel et al., 2017; Jepma \& Nieuwenhuis, 2011; Joshi et al., 2016), 


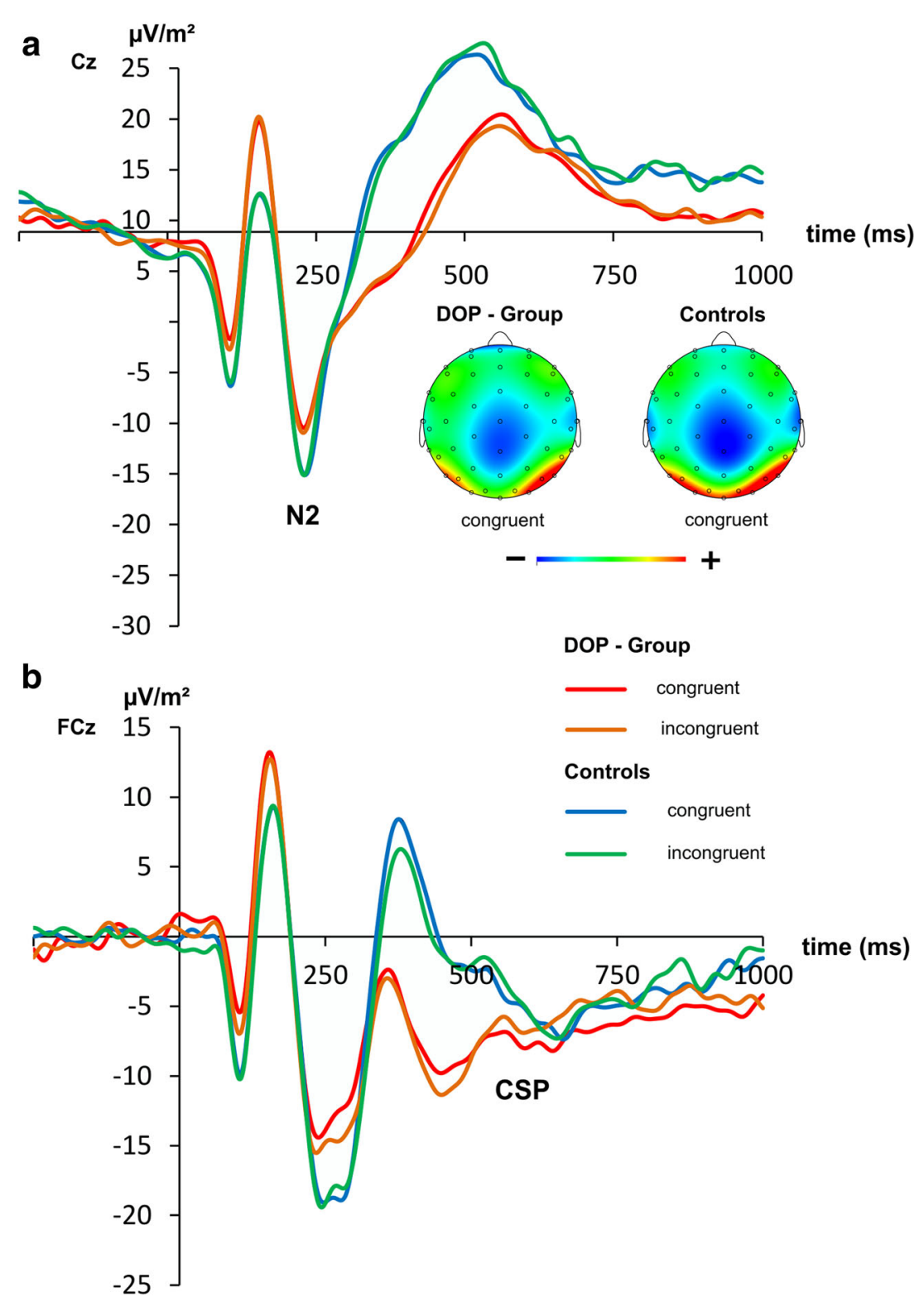

Fig. 4 (a) N2 ERP-component at electrode $\mathrm{Cz}$ and (b) conflict-slow potential at electrode $\mathrm{FCz}$ for each group (DOP group and controls). The corresponding topography maps of the scalp electrical potential are

it may be argued that these occur due to attentional processing of the P1 and N1 time windows. However, there was no interaction "congruency $\times$ group" in P1 and N1. Therefore, these correlations do not seem to reflect meaningful effects induced by the experimental manipulation and, therefore, cannot be interpreted.

As shown above, the correlations between the pupil diameter data and the ERP data in the DOP group were substantial. A correlation coefficient of $r>.5$ can be considered a "medium to strong" correlation (Evans, 1996). The obtained effect size for differential P3 amplitude effects was $\eta_{p}^{2}=.24$. With a sample size of $\mathrm{N}=20$ subjects and an alpha level of $\alpha=$ .05 , the achieved power, as calculated using the $G^{*}$ Power shown for the peak of N2- ERP component. In the topographies, red denotes positivity, and blue negativity. Time point zero denotes onset of trial stimulus. The lines represent congruent/incongruent emotion trials

software package, in this study is greater than $95 \%$. This shows that the study is sufficiently powered and that the obtained dissociation, i.e., lack of correlations between pupil diameter data and ERP data in the control group and the electrode sites reflecting attentional selection processes is reliable.

\section{Discussion}

The aim of the current study was to investigate the specific mechanisms underlying the effect of depth of processing (DOP) of emotional stimuli on the processing of emotional interference effects. We did so by integrating neurophysiological, 

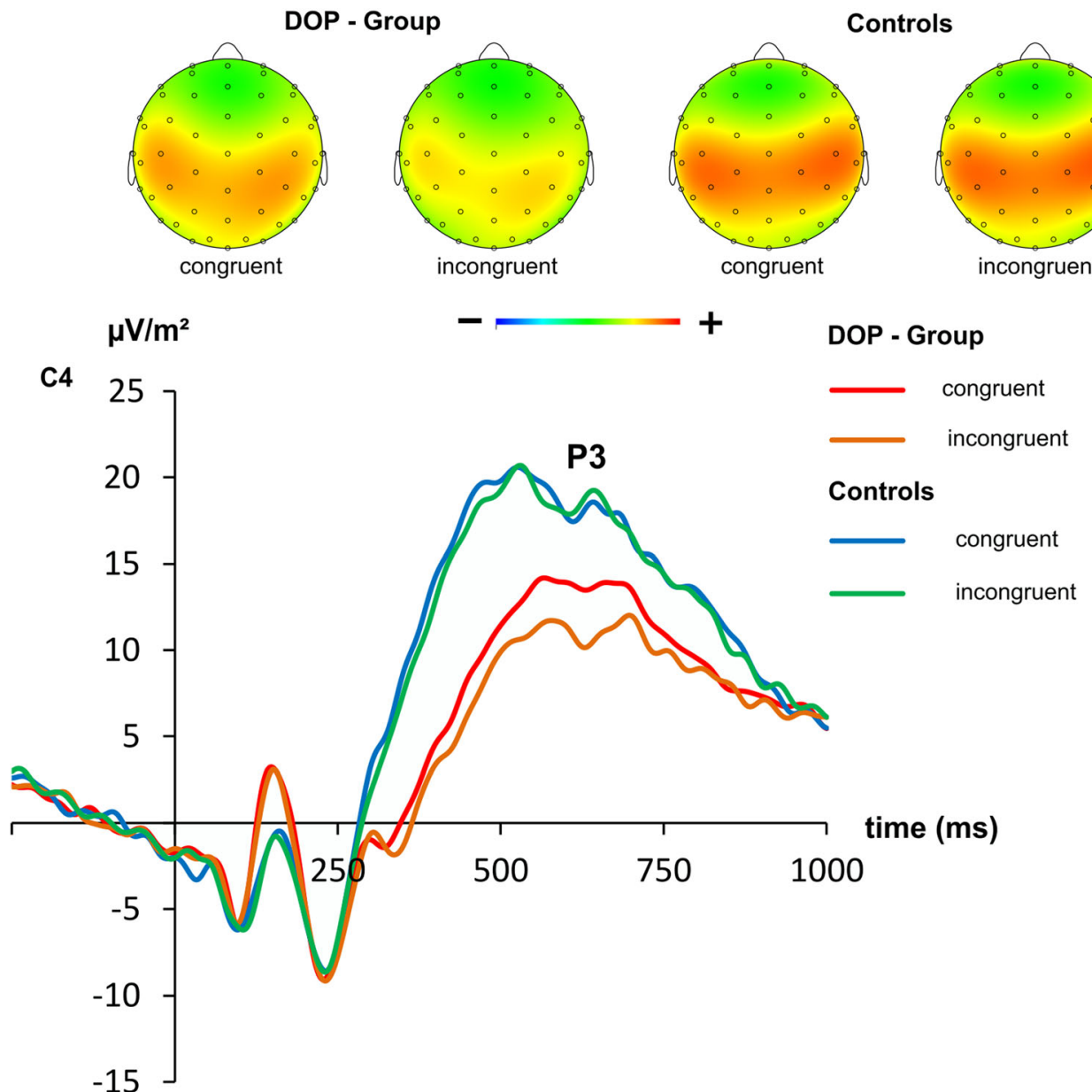

Controls

the target stimulus. There were significant differences between P3 amplitudes on congruent and incongruent trial in the DOP group, where the distracting stimulus dimension 2 required deeper levels of processing but not for the controls time-window (550-650 ms) for each group (DOP group and controls). In the topographies, red denotes positivity, and blue negativity. The lines represent congruent emotional trials. Time point zero denotes onset of

source localization, and pupil diameter data obtained from two groups of participants performing an emotional Stroop task. Overcoming emotional Stroop interference effects requires accurate and efficient processing of the task-relevant emotional stimulus dimension, while ignoring the distracting effects of a simultaneously presented task-irrelevant but still conflicting/ interfering emotional stimulus dimension. Importantly, the emotional Stroop effect, similar to non-emotional Stroop effects, represents a "stimulus-stimulus conflict" (S-S conflict) (Hommel, 2015). Therefore, we detailed how DOP affects neural mechanisms underlying this sort of conflict monitoring. We hypothesized that manipulating the DOP of the distractor stimulus dimension should increase emotional conflicts and should complicate decision-making processes regarding which response to select. These decision-making processes were expected to be modulated by the NE system in the DOP group, as indexed by correlations of the pupil diameter data and the EEG data in the $\mathrm{P} 3$ time window.
The behavioral results showed that emotional Stroop effects were stronger in the DOP group, in which the depth of processing of the irrelevant emotional word dimension of the Stroop stimulus was increased. Specifically, we found that within the DOP group, response accuracy was lower and response times slower compared to the control group. The emotional Stroop effect represents a "stimulus-stimulus conflict" (S-S conflict), which refers to competition and interference between two semantically similar stimulus dimensions that are simultaneously presented (Hommel, 2015). The intermittent queries within the DOP condition prompted participants in this group to allocate processing resources to the taskirrelevant stimulus dimension, which increases demands to resolve the emotional conflict compared to the control group. Thus, the behavioral data clearly shows that a manipulation of the DOP of irrelevant yet interfering emotional stimulus dimension constituting the S-S conflict modulates the strength of emotional conflicts. The neurophysiological data provide 
insights into what kind of cognitive mechanisms are affected by that, and revealed very specific effects.

We found no differential modulation explaining the behavioral data for N1 or P1 ERP-components. Thus, perceptual and early attentional processes reflected by these components are unlikely to underlie the differences observed on the behavioral level (Herrmann \& Knight, 2001). Interestingly, neither did the N2 ERP-component reflect the interactive effects on the behavioral level. With regard to the lack of N2-modulation it is important to consider that studies reporting $\mathrm{N} 2$ differences in response to conflict usually employ quite simple conflicting stimuli (e.g., Flanker or Simon paradigms). This allows faster conflict detection and resolution processes than complex emotional stimuli (Chen et al., 2016; Chmielewski \& Beste, 2017; Larson et al., 2014; Shang et al., 2016). This was to be expected, and is in line with previous investigations where we found no effects on N2 amplitude using this specific set of stimuli employed in other versions of the paradigm (Schreiter et al., 2018a, 2018b). Neither did we find interactive effects within the CSP data, which suggests that conflict monitoring or resolution processes in general are not modulated by varying the processing depth of emotional interfering stimuli. However, since results suggest that the CSP is mostly affected by pro-active control processes (Larson et al., 2014; Pan et al., 2016; Shen et al., 2013), this is an expected finding.

Importantly, interactive effects of congruency and group were observed for the P3 ERP-component. This is in line with our a priori hypothesis. The P3 component has consistently been found to be modulated in emotional Stroop conflict and tends to be stronger on emotionally congruent than incongruent trials, and more positive following negative compared to non-aversive/threatening emotion trials (Clayson \& Larson, 2013; Delplanque et al., 2004; Larson et al., 2014; Schreiter et al., 2018b; Yang et al., 2013). Our findings show that P3 modulations between congruent and incongruent emotional Stroop trials were stronger in the DOP group compared to the control group. Thus, P3 amplitude is reflective of the level of processing allocated to particular stimulus dimensions (Hong et al., 2014; Kok, 2001; Murphy et al., 2011). The P3 has been shown to reflect decision-making processes (Gray et al., 2004; Twomey et al., 2015) and has been found to be more enhanced with increasing demand for conflict control (Mennes et al., 2008). Likely, the P3 is modulated by the quantity of evidence required to trigger a decision (Twomey et al., 2015). Obviously, such a decision process becomes complicated by forcing participants in the DOP group to fully evaluate the task-relevant and the task-irrelevant dimension of the emotional Stroop stimulus. Notably, the results from the analysis of the pupil diameter data as an indirect index of the NE system activity are in line with the interpretation that decision processes are modulated by the manipulation of the DOP of emotionally conflicting stimuli. According to the adaptive-gain theory, the NE system plays a major role for more complex decision processes, and it has been suggested that the NE system facilitates neural processes at task-relevant decision points (AstonJones \& Cohen, 2005). Importantly, in the DOP group, the pupil diameter was selectively correlated with the neurophysiological data in the P3 time window. This strongly suggests that the NE system selectively modulates neural mechanisms during decision processes, which is in line with theoretical conceptions (Nieuwenhuis et al., 2005). It is well established that pupil reactions occur with an approximate delay of about $200 \mathrm{~ms}$ relative to LC-NE activity (Dippel et al., 2017; Jepma \& Nieuwenhuis, 2011; Joshi et al., 2016). Thus, the obtained positive correlations suggest that greater pupil diameter at a time point of around 700-1,000 ms post-stimulus onset is related to a larger/stronger P3 (at about 500-660 ms). However, even when assuming that this is not the case and the correlations fall after the P3 time window, this is still a reasonable finding because the late positive potentials are known to reflect affective picture processing (Hajcak \& Olvet, 2008), for which covariation with autonomic arousal has also been reported (Cuthbert, Schupp, Bradley, Birbaumer, \& Lang, 2000).

Larger pupil diameters have been shown to reflect higher NE concentrations (Hou et al., 2005b; Phillips et al., 2000). Therefore, a higher NE activity is associated with more demanding decision-making processes when the DOP of the irrelevant emotional distractor dimension is elevated. Crucially, modulations of neural processes by the NE system have been referred to as modulators of "gain control" (AstonJones \& Cohen, 2005). Elevated gain control facilitates information processing (Salinas \& Thier, 2000) and is evident at sensory and cognitive processing levels (Salinas \& Thier, 2000; Servan-Schreiber, Printz, \& Cohen, 1990). The obtained results are well in line with such gain-modulation mechanisms. Interestingly, several lines of evidence suggest that parietal areas in particular are subject to gain-control processes (Adelhöfer et al., 2018; Salinas \& Thier, 2000). In line with this, the sLORETA analysis showed that areas in the parietal cortex (BA7) and the insular cortex (BA13) were activated during the time period in which correlations between the pupil diameter data and the neurophysiological data were evident. This suggests that the NE system modulates complicated decision-making processes during emotional conflicts via a parietal-insular network. Interestingly, BA7 has recently been found to be modulated by gain-control processes under conditions of complex incoming sensory information (Adelhöfer et al., 2018). Several lines of evidence suggest an involvement of BA7 in cognitive control decision processes whenever information is complex and probably difficult to categorize but essential for behavioral control (Bodmer \& Beste, 2017; Bodmer, Mückschel, Roessner, \& Beste, 2018; Fokin et al., 2008; Ocklenburg, Güntürkün, \& Beste, 2011; Takeichi et al., 2010). Moreover, the precuneus (BA7) has been implicated in the processing of facial (emotional) information (Cheng et al., 2017; Kim et al., 2015; Zhang, Song, Liu, \& Liu, 2016; Zhao, 
a

pupil diameter

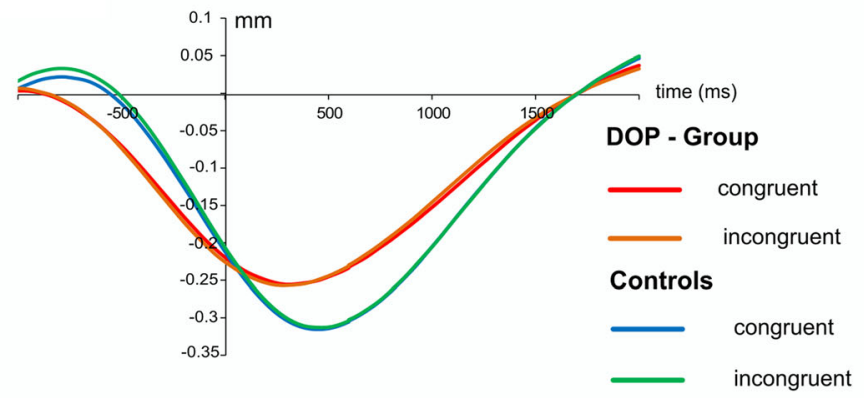

b
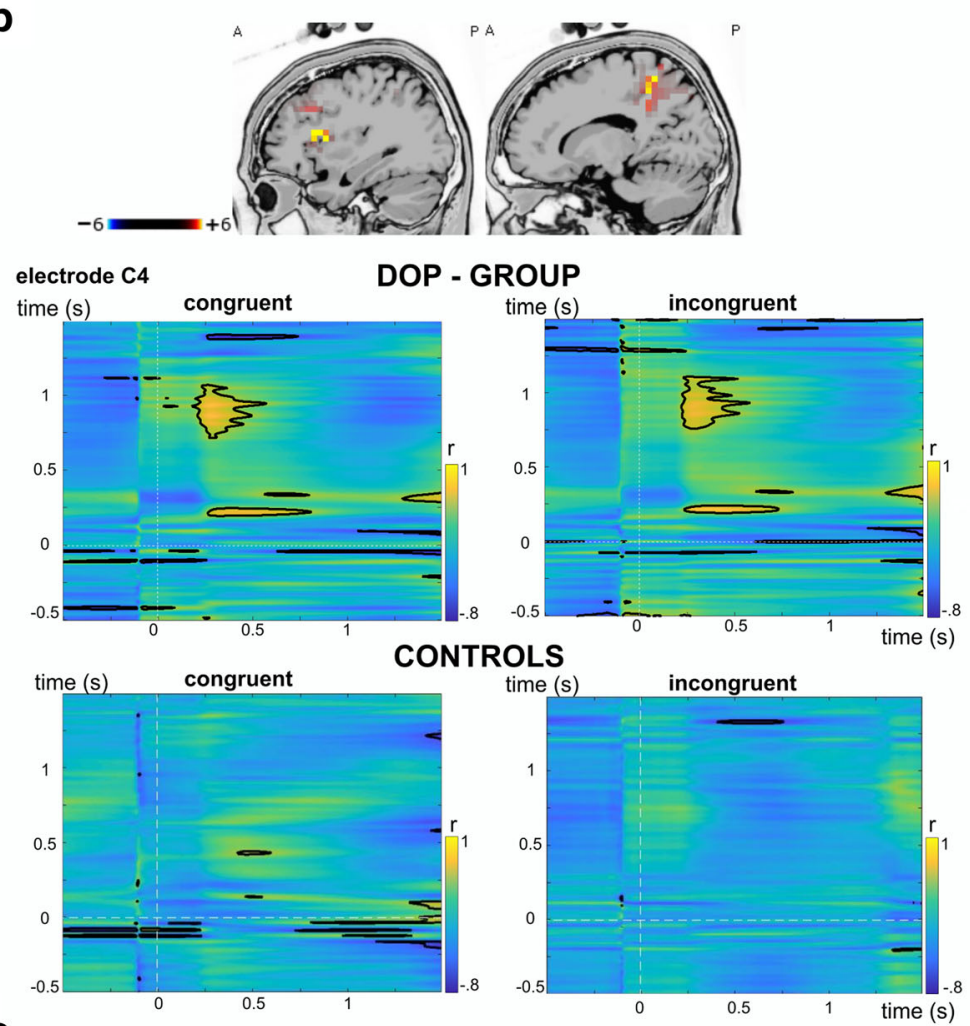

C
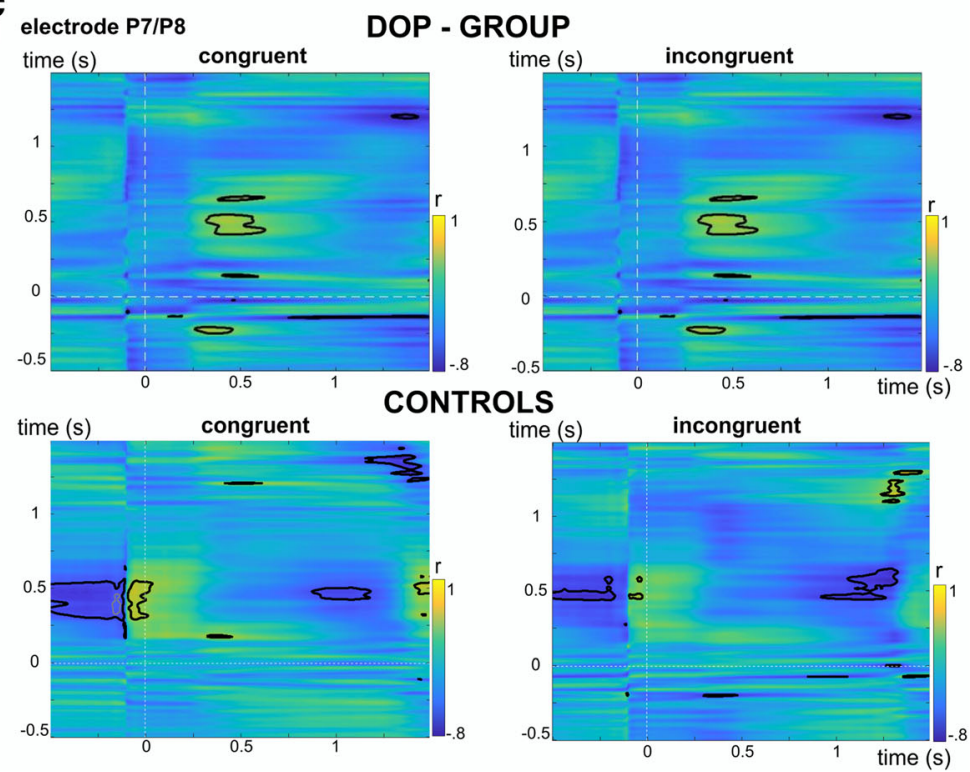
Fig. 6 Pupil diameter and EEG data integration and sLORETA results. Part a shows changes in pupil dilation (in $\mathrm{mm}$ ) as a function of time, for each group (DOP group/warm colors and controls/cool colors) for congruent and incongruent trials. Part b shows the correlation between time-specific changes within pupil diameter ( $\mathrm{x}$-axis) and the ERP time course at electrode $\mathrm{C} 4$ (y-axis) for each experimental group. The sLORETA plots show significant activation in the insular cortex (BA13) and the precuneus (BA7) for the time period in which correlations between the pupil diameter and the ERP data were evident. Part c shows correlation between time-specific changes within pupil diameter (x-axis) and P1/N1 electrodes P7/P8 (y-axis) for each experimental group. Significant correlations $(\mathrm{p}<.05)$ are highlighted by black outline, positive correlations are shown in yellow, and negative correlations are shown in blue

Zhao, Zhang, Cui, \& Fu, 2017). In the current experiment, the processing of the facial emotional dimension was complicated in the DOP group, because participants in this group were forced to evaluate the distracting semantic word information. It is therefore reasonable that functional neuroanatomical structures are involved, which are known to be activated whenever information is complex and probably difficult to categorize but essential for behavioral control. The insular cortex likely integrates sensory, cognitive, and emotional processes (Gogolla, 2017), plays a role in the interpretation of emotional information, and is involved in emotional and non-emotional interference processing (Gogolla, 2017; Simmons et al., 2008; Xue et al., 2016). It thus seems that the NE system modulates decision processes during emotional conflicts that are associated with functional neuroanatomical structures known to be important when information is complex and probably difficult to categorize but essential for informing behavioral control.

A limitation of the study is that possible interindividual differences regarding the processing of positive and negative emotions were not controlled for and examined. However, since the obtained effects revealed a reasonable effect size, it is unlikely that this has strongly biased effects. We would also like to acknowledge limitations concerning the sample characteristics that may have impacted on the results but were not explicitly controlled for in the current study. We did not collect data on individual different aspects of emotional wellbeing, intelligence, and socio-economic status of the participants. While it is possible that these factors influence the ability to overcome emotional conflict, it must be emphasized that both groups (DOP and control group) were randomly selected from a healthy and culturally homogenous student sample (German mother tongue, no psychiatric background, no regular medication) and, hence, any variations in the above-mentioned factors would have likely been there in either group. Thus, the group differences are most likely due to the existence of the query enhancing the relative importance of the distractor word and deeper processing of the stimuli in the DOP group. In this regard, a possible study limitation is that the control group was not confronted with infrequent queries unrelated to the emotional content of the distractor dimension. It is arguable, however, whether the addition of another unrelated question for the control group, would not induce more confounding effects (such as, switch costs), rather than control for the mere existence of a query. It would be interesting to include a control query in future studies.

An important future direction for the study of processing depth of emotional conflicting stimuli is related to the study design. In the current study, we employed a between-subject design in order to avoid effects or task order (learning effects) on the results. Though task-order effects could be ruled in data analyses, this would be interesting to investigate in future studies to further explore individual differences in DOP processing.

In summary, the study examined what cognitiveneurophysiological mechanisms are modulated when the depth of processing of emotional conflicting stimuli is varied. The study shows that when processing depth of conflicting stimuli is increased, emotional conflict effects become stronger. The EEG data show that this was associated with modulations of decision-making processes, as reflected by the P3 ERP-component. Notably, the integration with pupil diameter data suggests that these decision processes were modulated by the NE system, especially when the depth of processing of interfering emotional stimulus dimensions was increased. This likely reflects gain modulation processes in facilitating processing of complex interfering, emotional information. The source localization results suggest that regions in the parietal and insular cortex are associated with these modulatory effects.

Acknowledgements This work was partly supported by Grants from the Deutsche Forschungsgemeinschaft (DFG) BE4045/26-1 and the BMBF 01GL1741C.

\section{Compliance with ethical standards}

Open practices statement The data and materials for all experiments are available at https://osf.io/hm8gw/. The experiment was not preregistered.

\section{References}

Adelhöfer, N., Gohil, K., Passow, S., Teufert, B., Roessner, V., Li, S.-C., \& Beste, C. (2018). The system-neurophysiological basis for how methylphenidate modulates perceptual-attentional conflicts during auditory processing. Human Brain Mapping, 39(12), 5050-5061. https://doi.org/10.1002/hbm.24344

Algom, D., Chajut, E., \& Lev, S. (2004). A rational look at the emotional stroop phenomenon: a generic slowdown, not a stroop effect. Journal of Experimental Psychology. General, 133(3), 323-338. https://doi.org/10.1037/0096-3445.133.3.323

Aston-Jones, G., \& Cohen, J. D. (2005). Adaptive gain and the role of the locus coeruleus-norepinephrine system in optimal performance. The 
Journal of Comparative Neurology, 493(1), 99-110. https://doi.org/ 10.1002/cne.20723

Berg, H. E., Ballard, E. D., Luckenbaugh, D. A., Nugent, A. C., Ionescu, D. F., \& Zarate, C. A. (2016). Recognition of Emotional Facial Expressions in Anxious and Nonanxious Depression. Comprehensive Psychiatry, 70, 1-8. https://doi.org/10.1016/j.comppsych.2016.06.007

Bledowski, C., Prvulovic, D., Hoechstetter, K., Scherg, M., Wibral, M., Goebel, R., \& Linden, D. E. J. (2004). Localizing P300 Generators in Visual Target and Distractor Processing: A Combined EventRelated Potential and Functional Magnetic Resonance Imaging Study. Journal of Neuroscience, 24(42), 9353-9360. https://doi. org/10.1523/JNEUROSCI.1897-04.2004

Bodmer, B., \& Beste, C. (2017). On the dependence of response inhibition processes on sensory modality. Human Brain Mapping, 38(4), 1941-1951. https://doi.org/10.1002/hbm.23495

Bodmer, B., Mückschel, M., Roessner, V., \& Beste, C. (2018). Neurophysiological variability masks differences in functional neuroanatomical networks and their effectiveness to modulate response inhibition between children and adults. Brain Structure \& Function, 223(4), 1797-1810. https://doi.org/10.1007/s00429-017-1589-6

Brosch, T., Sander, D., Pourtois, G., \& Scherer, K. R. (2008). Beyond fear: rapid spatial orienting toward positive emotional stimuli. Psychological Science, 19(4), 362-370. https://doi.org/10.1111/j. 1467-9280.2008.02094.x

Chen, T., Kendrick, K. M., Feng, C., Sun, S., Yang, X., Wang, X., ... Luo, Y.-J. (2016). Dissociable early attentional control mechanisms underlying cognitive and affective conflicts. Scientific Reports, 6. https://doi.org/10.1038/srep37633

Cheng, W., Rolls, E. T., Zhang, J., Sheng, W., Ma, L., Wan, L., ... Feng, J. (2017). Functional connectivity decreases in autism in emotion, self, and face circuits identified by Knowledge-based Enrichment Analysis. NeuroImage, 148, 169-178. https://doi.org/10.1016/j. neuroimage.2016.12.068

Chmielewski, W. X., \& Beste, C. (2017). Testing interactive effects of automatic and conflict control processes during response inhibition - A system neurophysiological study. NeuroImage, 146, 1149 1156. https://doi.org/10.1016/j.neuroimage.2016.10.015

Chmielewski, W. X., Mückschel, M., Ziemssen, T., \& Beste, C. (2017). The norepinephrine system affects specific neurophysiological subprocesses in the modulation of inhibitory control by working memory demands. Human Brain Mapping, 38(1), 68-81. https://doi.org/ $10.1002 / \mathrm{hbm} .23344$

Clayson, P. E., \& Larson, M. J. (2013). Adaptation to Emotional Conflict: Evidence from a Novel Face Emotion Paradigm. PLOS ONE, 8(9), e75776. https://doi.org/10.1371/journal.pone.0075776

Costa, V. D., \& Rudebeck, P. H. (2016). More than Meets the Eye: the Relationship between Pupil Size and Locus Coeruleus Activity. Neuron, 89(1), 8-10. https://doi.org/10.1016/j.neuron.2015.12.031

Cuthbert, B. N., Schupp, H. T., Bradley, M. M., Birbaumer, N., \& Lang, P. J. (2000). Brain potentials in affective picture processing: covariation with autonomic arousal and affective report. Biological Psychology, 52(2), 95-111.

Delorme, A., \& Makeig, S. (2004). EEGLAB: an open source toolbox for analysis of single-trial EEG dynamics including independent component analysis. Journal of Neuroscience Methods, 134(1), 9-21. https://doi.org/10.1016/j.jneumeth.2003.10.009

Delplanque, S., Lavoie, M. E., Hot, P., Silvert, L., \& Sequeira, H. (2004). Modulation of cognitive processing by emotional valence studied through event-related potentials in humans. Neuroscience Letters, 356(1), 1-4. https://doi.org/10.1016/j.neulet.2003.10.014

Dimigen, O., Sommer, W., Hohlfeld, A., Jacobs, A. M., \& Kliegl, R. (2011). Coregistration of eye movements and EEG in natural reading: analyses and review. Journal of Experimental Psychology. General, 140(4), 552-572. https://doi.org/10.1037/a0023885

Dippel, G., \& Beste, C. (2015). A causal role of the right inferior frontal cortex in implementing strategies for multi-component behaviour.
Nature Communications, 6, 6587. https://doi.org/10.1038/ ncomms 7587

Dippel, G., Mückschel, M., Ziemssen, T., \& Beste, C. (2017). Demands on response inhibition processes determine modulations of theta band activity in superior frontal areas and correlations with pupillometry - Implications for the norepinephrine system during inhibitory control. NeuroImage, 157, 575-585. https://doi.org/10. 1016/j.neuroimage.2017.06.037

Dosher, B. A., \& Lu, Z. L. (1998). Perceptual learning reflects external noise filtering and internal noise reduction through channel reweighting. Proceedings of the National Academy of Sciences of the United States of America, 95(23), 13988-13993.

Egner, T., Etkin, A., Gale, S., \& Hirsch, J. (2008). Dissociable Neural Systems Resolve Conflict from Emotional versus Nonemotional Distracters. Cerebral Cortex, 18(6), 1475-1484. https://doi.org/10. 1093/cercor/bhm179

Etkin, A., Egner, T., \& Kalisch, R. (2011). Emotional processing in anterior cingulate and medial prefrontal cortex. Trends in Cognitive Sciences, 15(2), 85-93. https://doi.org/10.1016/j.tics.2010.11.004

Evans, J. D. (1996). Straightforward Statistics for the Behavioral Sciences. Brooks/Cole Publishing Company.

Fokin, V. A., Shelepin, Y. E., Kharauzov, A. K., Trufanov, G. E., Sevost'yanov, A. V., Pronin, S. V., \& Koskin, S. A. (2008). Localization of human cortical areas activated on perception of ordered and chaotic images. Neuroscience and Behavioral Physiology, 38(7), 677-685. https://doi.org/10.1007/s11055-008-9033-2

Gogolla, N. (2017). The insular cortex. Current Biology: CB, 27(12), R580-R586. https://doi.org/10.1016/j.cub.2017.05.010

Gray, H. M., Ambady, N., Lowenthal, W. T., \& Deldin, P. (2004). P300 as an index of attention to self-relevant stimuli. Journal of Experimental Social Psychology, 40(2), 216-224. https://doi.org/ 10.1016/S0022-1031(03)00092-1

Hajcak, G., \& Olvet, D. M. (2008). The persistence of attention to emotion: brain potentials during and after picture presentation. Emotion (Washington, D.C.), 8(2), 250-255. https://doi.org/10.1037/15283542.8.2.250

Helton, W. S., Hollander, T. D., Warm, J. S., Matthews, G., Dember, W. N., Wallaart, M., ... Hancock, P. A. (2005). Signal regularity and the mindlessness model of vigilance. British Journal of Psychology (London, England: 1953), 96(Pt 2), 249-261. https://doi.org/10. 1348/000712605X38369

Herrmann, C. S., \& Knight, R. T. (2001). Mechanisms of human attention: event-related potentials and oscillations. Neuroscience and Biobehavioral Reviews, 25(6), 465-476.

Hoffmann, S., Labrenz, F., Themann, M., Wascher, E., \& Beste, C. (2014). Crosslinking EEG time-frequency decomposition and fMRI in error monitoring. Brain Structure \& Function, 219(2), 595-605. https://doi.org/10.1007/s00429-013-0521-y

Hommel, B. (2015). The theory of event coding (TEC) as embodiedcognition framework. Frontiers in Psychology, 6. https://doi.org/ 10.3389/fpsyg.2015.01318

Hong, L., Walz, J. M., \& Sajda, P. (2014). Your Eyes Give You Away: Prestimulus Changes in Pupil Diameter Correlate with Poststimulus Task-Related EEG Dynamics. PLOS ONE, 9(3), e91321. https://doi. org/10.1371/journal.pone.0091321

Hou, R. H., Freeman, C., Langley, R. W., Szabadi, E., \& Bradshaw, C. M. (2005). Does modafinil activate the locus coeruleus in man? Comparison of modafinil and clonidine on arousal and autonomic functions in human volunteers. Psychopharmacology, 181(3), 537549. https://doi.org/10.1007/s00213-005-0013-8

Jepma, M., \& Nieuwenhuis, S. (2011). Pupil diameter predicts changes in the exploration-exploitation trade-off: evidence for the adaptive gain theory. Journal of Cognitive Neuroscience, 23(7), 1587-1596. https://doi.org/10.1162/jocn.2010.21548

Joshi, S., Li, Y., Kalwani, R. M., \& Gold, J. I. (2016). Relationships between Pupil Diameter and Neuronal Activity in the Locus 
Coeruleus, Colliculi, and Cingulate Cortex. Neuron, 89(1), 221234. https://doi.org/10.1016/j.neuron.2015.11.028

Kayser, J., \& Tenke, C. E. (2015). On the benefits of using surface Laplacian (current source density) methodology in electrophysiology. International Journal of Psychophysiology: Official Journal of the International Organization of Psychophysiology, 97(3), 171173. https://doi.org/10.1016/j.ijpsycho.2015.06.001

Kerns, J. G., Cohen, J. D., MacDonald, A. W., Cho, R. Y., Stenger, V. A., \& Carter, C. S. (2004). Anterior cingulate conflict monitoring and adjustments in control. Science (New York, N.Y.), 303(5660), 10231026. https://doi.org/10.1126/science.1089910

Kim, J., Schultz, J., Rohe, T., Wallraven, C., Lee, S.-W., \& Bülthoff, H. H. (2015). Abstract representations of associated emotions in the human brain. The Journal of Neuroscience: The Official Journal of the Society for Neuroscience, 35(14), 5655-5663. https://doi.org/10. 1523/JNEUROSCI.4059-14.2015

Kok, A. (2001). On the utility of P3 amplitude as a measure of processing capacity. Psychophysiology, 38(3), 557-577.

Kunde, W., \& Mauer, N. (2008). Sequential Modulations of Valence Processing in the Emotional Stroop Task. Experimental Psychology, 55(3), 151-156. https://doi.org/10.1027/1618-3169. 55.3.151

Larson, M. J., Clayson, P. E., \& Clawson, A. (2014). Making sense of all the conflict: a theoretical review and critique of conflict-related ERPs. International Journal of Psychophysiology: Official Journal of the International Organization of Psychophysiology, 93(3), 283297. https://doi.org/10.1016/j.ijpsycho.2014.06.007

Ma, J., Liu, C., Zhong, X., Wang, L., \& Chen, X. (2014). Emotional Body-Word Conflict Evokes Enhanced N450 and Slow Potential. PLOS ONE, 9(5), e95198. https://doi.org/10.1371/journal.pone. 0095198

MacLeod, C. M. (1991). Half a century of research on the Stroop effect: an integrative review. Psychological Bulletin, 109(2), 163-203.

Marco-Pallarés, J., Grau, C., \& Ruffini, G. (2005). Combined ICALORETA analysis of mismatch negativity. NeuroImage, 25(2), 471-477. https://doi.org/10.1016/j.neuroimage.2004.11.028

Marusak, H. A., Martin, K. R., Etkin, A., \& Thomason, M. E. (2015). Childhood trauma exposure disrupts the automatic regulation of emotional processing. Neuropsychopharmacology: Official Publication of the American College of Neuropsychopharmacology, 40(5), 12501258. https://doi.org/10.1038/npp.2014.311

McKenna, F. P., \& Sharma, D. (2004). Reversing the Emotional Stroop Effect Reveals That It Is Not What It Seems: The Role of Fast and Slow Components. Journal of Experimental Psychology: Learning, Memory, and Cognition, 30(2), 382-392. https://doi.org/10.1037/ 0278-7393.30.2.382

Mennes, M., Wouters, H., Bergh, B. V. D., Lagae, L., \& Stiers, P. (2008). ERP correlates of complex human decision making in a gambling paradigm: Detection and resolution of conflict. Psychophysiology, 45(5), 714-720. https://doi.org/10.1111/j.1469-8986.2008.00678.x

Mückschel, M., Chmielewski, W., Ziemssen, T., \& Beste, C. (2017a). The norepinephrine system shows information-content specific properties during cognitive control - Evidence from EEG and pupillary responses. NeuroImage, 149, 44-52. https://doi.org/10.1016/j. neuroimage.2017.01.036

Mückschel, M., Gohil, K., Ziemssen, T., \& Beste, C. (2017b). The norepinephrine system and its relevance for multi-component behavior. NeuroImage, 146, 1062-1070. https://doi.org/10.1016/j. neuroimage.2016.10.007

Mückschel, M., Stock, A.-K., \& Beste, C. (2014). Psychophysiological mechanisms of interindividual differences in goal activation modes during action cascading. Cerebral Cortex (New York, N.Y.: 1991), 24(8), 2120-2129. https://doi.org/10.1093/cercor/bht066

Murphy, P. R., Robertson, I. H., Balsters, J. H., \& O'connell, R. G. (2011). Pupillometry and P3 index the locus coeruleusnoradrenergic arousal function in humans. Psychophysiology,
48(11), 1532-1543. https://doi.org/10.1111/j.1469-8986.2011. 01226.x

Nieuwenhuis, S., De Geus, E. J., \& Aston-Jones, G. (2011). The anatomical and functional relationship between the $\mathrm{P} 3$ and autonomic components of the orienting response. Psychophysiology, 48(2), 162175. https://doi.org/10.1111/j.1469-8986.2010.01057.x

Nieuwenhuis, S., Heslenfeld, D. J., von Geusau, N. J. A., Mars, R. B., Holroyd, C. B., \& Yeung, N. (2005). Activity in human rewardsensitive brain areas is strongly context dependent. NeuroImage, 25(4), 1302-1309.

Nunez, P. L., \& Pilgreen, K. L. (1991). The Spline-Laplacian in Clinical Neurophysiology: A Method to Improve EEG Spatial Resolution. Journal of Clinical Neurophysiology, 8(4), 397-413. https://doi.org/ 10.1097/00004691-199110000-00005

Ochsner, K. N., \& Gross, J. J. (2005). The cognitive control of emotion. Trends in Cognitive Sciences, 9(5), 242-249. https://doi.org/10. 1016/j.tics.2005.03.010

Ocklenburg, S., Güntürkün, O., \& Beste, C. (2011). Lateralized neural mechanisms underlying the modulation of response inhibition processes. NeuroImage, 55(4), 1771-1778. https://doi.org/10.1016/j. neuroimage.2011.01.035

Pan, F., Lu, Q., Chen, Y., Wu, X., \& Li, Q. (2016). Emotional conflict occurs at a late stage: evidence from the paired-picture paradigm. Translational Neuroscience, 7(1), 56-61. https://doi.org/10.1515/ tnsci-2016-0009

Pascual-Marqui, R. D. (2002). Standardized low-resolution brain electromagnetic tomography (sLORETA): technical details. Methods and Findings in Experimental and Clinical Pharmacology, 24 Suppl D, 5-12.

Phillips, M. A., Szabadi, E., \& Bradshaw, C. M. (2000). Comparison of the effects of clonidine and yohimbine on spontaneous pupillary fluctuations in healthy human volunteers. Psychopharmacology, $150(1), 85-89$

Pool, E., Brosch, T., Delplanque, S., \& Sander, D. (2016). Attentional bias for positive emotional stimuli: A meta-analytic investigation. Psychological Bulletin, 142(1), 79-106. https://doi.org/10.1037/ bul0000026

Salinas, E., \& Thier, P. (2000). Gain Modulation: A Major Computational Principle of the Central Nervous System. Neuron, 27(1), 15-21. https://doi.org/10.1016/S0896-6273(00)00004-0

Saunders, B., Milyavskaya, M., \& Inzlicht, M. (2015). Variation in Cognitive Control as Emotion Regulation. Psychological Inquiry, 26(1), 108-115. https://doi.org/10.1080/1047840X.2015.962396

Schreiter, M. L., Chmielewski, W., \& Beste, C. (2018a). Neurophysiological processes and functional neuroanatomical structures underlying proactive effects of emotional conflicts. NeuroImage, 174, 11-21. https://doi.org/10.1016/j.neuroimage. 2018.03.017

Schreiter, M. L., Chmielewski, W. X., \& Beste, C. (2018b). How socioemotional setting modulates late-stage conflict resolution processes in the lateral prefrontal cortex. Cognitive, Affective, \& Behavioral Neuroscience, 1-15. https://doi.org/10.3758/s13415018-0585-5

Sekihara, K., Sahani, M., \& Nagarajan, S. S. (2005). Localization bias and spatial resolution of adaptive and non-adaptive spatial filters for MEG source reconstruction. NeuroImage, 25(4), 1056-1067. https://doi.org/10.1016/j.neuroimage.2004.11.051

Servan-Schreiber, D., Printz, H., \& Cohen, J. D. (1990). A network model of catecholamine effects: gain, signal-to-noise ratio, and behavior. Science (New York, N.Y.), 249(4971), 892-895.

Shang, Q., Fu, H., Qiu, W., \& Ma, Q. (2016). Event-related lateralized readiness potential correlates of the emotion-priming Simon effect. Experimental Brain Research, 234(8), 2123-2132. https://doi.org/ 10.1007/s00221-016-4614-8

Shen, Y., Xue, S., Wang, K., \& Qiu, J. (2013). Neural time course of emotional conflict control: an ERP study. Neuroscience Letters, 541, 34-38. https://doi.org/10.1016/j.neulet.2013.02.032 
Simmons, A., Matthews, S. C., Paulus, M. P., \& Stein, M. B. (2008). Intolerance of uncertainty correlates with insula activation during affective ambiguity. Neuroscience Letters, 430(2), 92-97. https:// doi.org/10.1016/j.neulet.2007.10.030

Takeichi, H., Koyama, S., Terao, A., Takeuchi, F., Toyosawa, Y., \& Murohashi, H. (2010). Comprehension of degraded speech sounds with m-sequence modulation: an fMRI study. NeuroImage, 49(3), 2697-2706. https://doi.org/10.1016/j.neuroimage.2009.10.063

Twomey, D. M., Murphy, P. R., Kelly, S. P., \& O'Connell, R. G. (2015). The classic $\mathrm{P} 300$ encodes a build-to-threshold decision variable. The European Journal of Neuroscience, 42(1), 1636-1643. https://doi. org/10.1111/ejn. 12936

Varazzani, C., San-Galli, A., Gilardeau, S., \& Bouret, S. (2015). Noradrenaline and Dopamine Neurons in the Reward/Effort TradeOff: A Direct Electrophysiological Comparison in Behaving Monkeys. Journal of Neuroscience, 35(20), 7866-7877. https:// doi.org/10.1523/JNEUROSCI.0454-15.2015

Warren, C. M., \& Holroyd, C. B. (2012). The Impact of Deliberative Strategy Dissociates ERP Components Related to Conflict Processing vs. Reinforcement Learning. Frontiers in Neuroscience, 6, 43. https://doi.org/10.3389/fnins.2012.00043

Weissman, D. H., \& Carp, J. (2013). Congruency sequence effects are driven by previous-trial congruency, not previous-trial response conflict. Frontiers in Psychology, 4. https://doi.org/10.3389/fpsyg.2013. 00587

Wolff, N., Mückschel, M., Ziemssen, T., \& Beste, C. (2018). The role of phasic norepinephrine modulations during task switching: evidence for specific effects in parietal areas. Brain Structure \& Function, 223(2), 925-940. https://doi.org/10.1007/s00429-017-1531-y

Xue, S., Li, Y., Kong, X., He, Q., Liu, J., \& Qiu, J. (2016). The dissociable neural dynamics of cognitive conflict and emotional conflict control: An ERP study. Neuroscience Letters, 619, 149-154. https://doi.org/ 10.1016/j.neulet.2016.03.020

Yang, J., Zeng, J., Meng, X., Zhu, L., Yuan, J., Li, H., \& Yusoff, N. (2013). Positive words or negative words: Whose valence strength are we more sensitive to? Brain Research, 1533, 91-104. https://doi. org/10.1016/j.brainres.2013.08.020

Zhang, L., Song, Y., Liu, L., \& Liu, J. (2016). Dissociable roles of internal feelings and face recognition ability in facial expression decoding. NeuroImage, 132, 283-292. https://doi.org/10.1016/j.neuroimage. 2016.02.049

Zhao, K., Zhao, J., Zhang, M., Cui, Q., \& Fu, X. (2017). Neural Responses to Rapid Facial Expressions of Fear and Surprise. Frontiers in Psychology, 8, 761. https://doi.org/10.3389/fpsyg. 2017.00761

Zinchenko, A., Kanske, P., Obermeier, C., Schröger, E., \& Kotz, S. A. (2015). Emotion and goal-directed behavior: ERP evidence on cognitive and emotional conflict. Social Cognitive and Affective Neuroscience, 10(11), 1577-1587. https://doi.org/10.1093/scan/ nsv050

Publisher's note Springer Nature remains neutral with regard to jurisdictional claims in published maps and institutional affiliations. 